\title{
Novel Shampoo Reduces Hair Shedding by Contracting the Arrector Pili Muscle via the Trace Amine Associate Receptor
}

Maja Kovacevic, John McCoy*, Andy Goren, Mirna Situm, Andrija Stanimirovic, Liu Wei, Yimei Tan, Sergio Vaño-Galvan, Jerry Shapiro, and Rodney Sinclair

*Author whom correspondence should be addressed (johnm@appliedbiology.com)

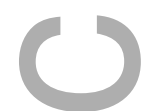

\section{Author Affiliations:}

Maja Kovacevic, MD: Department of Dermatology and Venereology, University of Rome "G.Marconi", Rome, Italy.

John McCoy, PhD: Applied Biology, Irvine, California, USA.

Andy Goren, MD: Applied Biology, Irvine, California, USA. Department of

Dermatology and Venereology, University of Rome "G.Marconi", Italy.

Mirna Situm, MD: Department of Dermatology and Venereology, University Hospital Center "Sestre milosrdnice", Zagreb, Croatia.

Andrija Stanimirovic, MD, PhD: Department of Clinical Medicine, University of Applied Health Sciences, Zagreb, Croatia

Liu Wei, MD: General Hospital of Air Force, PLA, Beijing, China.

Yimei Tan, MD: Skin \& Cosmetic Research Department, Shanghai Skin Disease Hospital, Shanghai, China.

This is the author manuscript accepted for publication and has undergone full peer review but has not been through the copyediting, typesetting, pagination and proofreading process, which may lead to differences between this version and the Version of Record. Please cite this article as doi: 10.1111/jocd.13054

This article is protected by copyright. All rights reserved 
Sergio Vaño-Galvan, MD, PHD. Trichology Unit, Dermatology Department, Ramon y Cajal Hospital, IRYCIS, University of Alcala, Madrid, Spain.

Jerry Shapiro, MD: Department of Dermatology, New York University Langone Medical Center, New York City, New York, USA.

Rodney Sinclair, MD: Department of Medicine, University of Melbourne, Victoria, Australia.
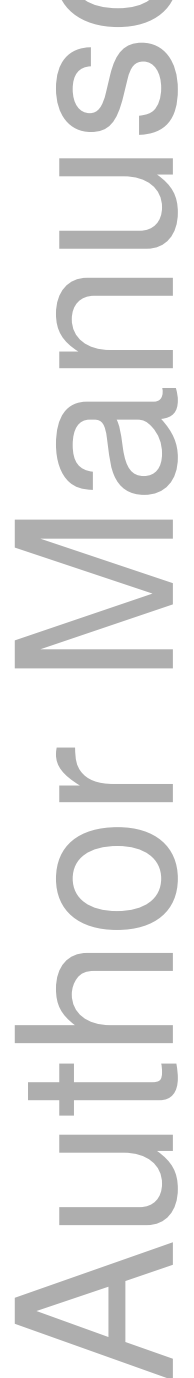
DR. J. MCCOY (Orcid ID : 0000-0003-1577-9910)

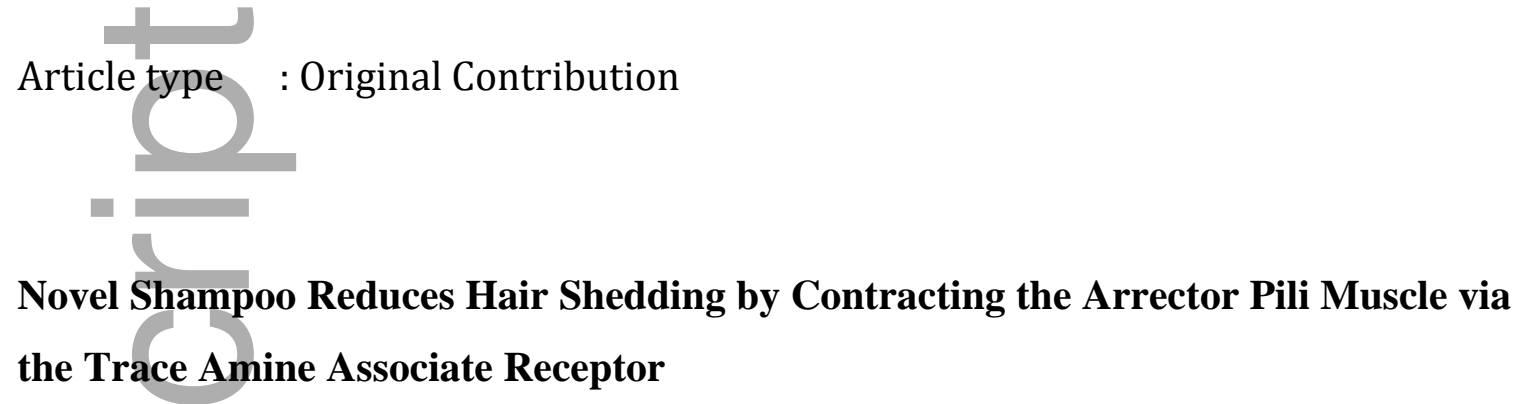

g

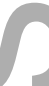

ABSTRACT

Background: Approximately $40 \%$ of women experience excessive hair shedding when washing their hair. Previously, we have demonstrated that a topically applied $\alpha 1$ adrenergic receptor agonist can be used to contract the arrector pili muscle of the follicular unit (i.e., produce "goose bumps"), increasing the force required to pluck hair by as much as $400 \%$. Subsequently, we reported a topical cosmetic solution containing an $\alpha 1$ adrenergic receptor agonist that reduced hair shedding during brushing by a maximum of $77 \%$.

Aims: In this communication we explore a novel mechanism to contract the arrector pili muscle. Trace amine associated receptors (TAAR) have been shown to regulate smooth muscle tone in blood vessels, but have not been reported to be present in the skin. Here we report on the anti-shedding efficacy of a shampoo containing a selective TAAR agonist, tyramine hydrochloride.

Methods: A single-blind cross over study was designed to test the efficacy of the novel shampoo versus placebo in reducing hairs lost during brushing.

Results: In this study, the novel TAAR shampoo reduced hair shedding during brushing by $31 \%$ in a cohort of 24 women with a maximum reduction of $77 \%$.

\section{INTRODUCTION}

This article is protected by copyright. All rights reserved 
Hair shedding is a normal part of the physiological processes of the body. Normally, women shed between 50 to 150 hairs in a 24-h period, while shedding in excess of 150 hairs can indicate thinning (1). In a survey of 300 women visiting a public hospital for conditions other than alopecia, approximately $40 \%$ reported experiencing excessive hair shedding subsequent to hair washing (as defined by the Sinclair Hair Shedding Scale) (2, 3). Increased shedding in women can sometimes be attributed to underlying medical conditions (4), however, even in healthy individuals many common hair styling practices can lead to excess shedding. For example, blow drying, flat ironing, hair curling, and brushing all apply traumatic force to hair follicles and consequently remove hair.

Hair follicles in the scalp are connected to the inner surface of the basal epidermis by smooth muscle, i.e., the arrector pili muscle. Its contraction produces piloerection, which is characterized by the phenotypical puckering of the skin around the hair or "goose bump". Piloerection can be stimulated by signaling molecules binding to receptors present on the arrector pili muscle (5). The force applied to the hair shaft during contraction of the arrector pili can greatly increases the threshold of force required to pluck hair.

Previously, we have reported on two molecules known to be agonists of the $\alpha_{1}$ adrenergic receptor $\left(\alpha_{1}-\mathrm{AR}\right)$, a G-protein coupled receptor, expressed on the arrector pili muscle. In a pilot study of 15 female subjects (6), we demonstrated that topically applying the $\alpha_{1}$ adrenergic receptor agonist, phenylephrine hydrochloride, increased the average threshold of force required to pluck hair by $172 \%$. Additionally, topical phenylephrine reduced shedding during brushing by $40 \%$. In a follow up study (7), we demonstrated a topical formula containing synephrine hydrochloride (AB-102) had similar efficacy. AB102 reduced the number of hairs lost during brushing by $38 \%$ in a cohort of 40 women with a maximum reduction of $77 \%$. Furthermore, the threshold force for epilation was increased by a maximum of $86 \%$. During both studies, no cardiac or hemodynamic changes were observed in any of the subjects sampled. 
Trace amine-associated receptors (TAARs) are a different class of G protein-coupled receptors. TAARs bind trace amines found naturally in mammals, for example, phenylethylamine, tyramine, and tryptamine (8). Recently it has been reported that TAARs are located in vascular smooth muscle and can be stimulated to cause smooth muscle tone changes independent of the sympathetic mechanism, e.g., $\alpha_{1}$-AR (9). We hypothesized that a TAAR agonist could be used to induce piloerection and increase the threshold for epilation similar to the $\alpha_{1}$-AR agonists we have previously studied. The objective of this study was to evaluate the effectiveness of a novel shampoo containing tyramine hydrochloride, a selective TAAR agonist, in reducing hair shedding in women.

\section{MATERIALS AND METHODS}

Twenty-four healthy female subjects, ages 18-65, were recruited from an outpatient dermatology clinic at (Blinded for peer review) Hospital. Subjects were recruited based on complaint of excessive hair shedding subsequent to hair washing. Subjects with uncontrolled hypertension, that were pregnant or breastfeeding, had been diagnosed with pattern hair loss or with other hair loss in conjunction with female pattern hair loss were excluded from the study. The study was conducted with the approval of the hospital ethics committee. All subjects gave informed consent during enrolment in the study.

To measure hair loss after hair washing, a 4-day protocol was designed. On the first day patients were instructed to wash their hair and use styling products as they normally would. On the second day, patients were instructed to wash their hair with a placebo shampoo, containing the vehicle only. Subsequent to hair washing, patients were instructed to brush their hair 20 times from the front of the scalp to the bottom of head using a new brush. After the procedure, the brushes were placed in a labeled plastic bag. On day three, patients were instructed to wash their hair and use styling products as they normally would. On the forth day, patients repeated the procedures of day two; only they applied the novel TAAR agonist shampoo. At the end of the study, the investigator counted the hairs collected on each brush. 


\section{RESULTS}

Twenty-four subjects completed the study. The raw data is tabulated in Table 1. The paired sample t-test was used to determine the effect of the novel TAAR shampoo compared to the vehicle shampoo on hair shedding subsequent to hair washing. The mean hair shedding in the TAAR shampoo group was $19.38 \pm 20.58$ compared to $27.92 \pm$ 22.36 in the placebo group. The average reduction in hairs lost during brushing after using the TAAR shampoo versus placebo was $31 \%$ for the cohort with a maximum reduction of $77 \%$. The analysis of the dataset (MedCalc v18.2.1) yielded a paired sample t-test value of 4.012 ( $\mathrm{p}=0.005)$; indicating that the use of the novel TAAR shampoo resulted in significant reduction in hair shedding subsequent to hair washing compared to the vehicle shampoo.

\section{DISCUSSION}

A significant amount of women report excessive shedding after washing their hair (3). To-date, no treatment is available for the reduction of hair shedding caused by the mechanical stress of everyday washing and styling. Previously, our group demonstrated that a solution containing synephrine hydrocholride significantly reduced hair shedding during brushing (7). Unfortunately, due to the high concentration of the weak agonist required to affect the arrector pili muscles in hair follicles, development of a shampoo with synephrine hydrochloride was not feasible. As such, we set to discover an alternate mechanism to contract the arrector pili muscle in hair follicles. This led us to hypothesize that TAAR receptors might be present in skin.

In this study, a shampoo formulated with a selective TAAR agonist was demonstrated to contract the arrector pili muscle and reduce hair shedding subsequent to washing. These data indirectly provide evidence of the expression of TAAR receptors in the arrector pili muscle. Additionally, the TAAR agonist shampoo showed similar efficacy to the synephrine hydrocholride solution previously tested (7) in reducing shedding during brushing. The average reduction was $31 \%$ for the cohort with a 
maximum reduction of $77 \%$. We hope this novel discovery will enable the development of future products to reduce excessive hair shedding.

\section{REFERENCES}

1. Sinclair R. Diffuse hair loss. International Journal of Dermatology 1999; 38: 8-18.

2. Sinclair R. Hair shedding in women: how much is too much? British Journal of Dermatology 2015; 173(3): 846-848.

3. Kovacevic M, Goren A, Shapiro J, Sinclair R, Lonky NM, Situm M, Bulat V, Bolanca Z, McCoy J. Prevalence of hair shedding among women. Dermatologic Therapy 2017; 30(1): e12415.

4. Malkud S. Telogen effluvium: A review. Journal of Clinical and Diagnostic Research 2015; 9: WE01-WE03.

5. Siepmann T, Gibbons CH, Illigens BM, Lafo JA, Brown CM, Freeman R. Quantitative pilomotor axon reflex test: A novel test of pilomotor function. Archives of Neurology 2012; 69(11): 1488-1492.

6. Goren A, Shapiro J, Sinclair R, Kovacevic M, McCoy J. a1-AR agonist induced piloerection protects against the development of traction alopecia. Dermatologic Therapy 2016; 29: 160-163.

7. McCoy J, Goren A, Kovacevic M, Situm M, Stanimirovic A, Shapiro J, Sinclair R. Styling without shedding: Novel topical formula reduces hair shedding by contracting the arrector pili muscle. Dermatologic Therapy 2017; e12575.

8. Broadley KJ. The vascular effects of trace amines and amphetamines. Pharmacology \& Therapeutics 2010; 125: 363-375.

9. Broadley KJ, Fehler M, Ford WR, Kidd EJ. Functional evaluation of the receptors mediating vasoconstriction of rat aorta by trace amines and amphetamines. European Journal of Pharmacology 2013; 715: 370-380. 


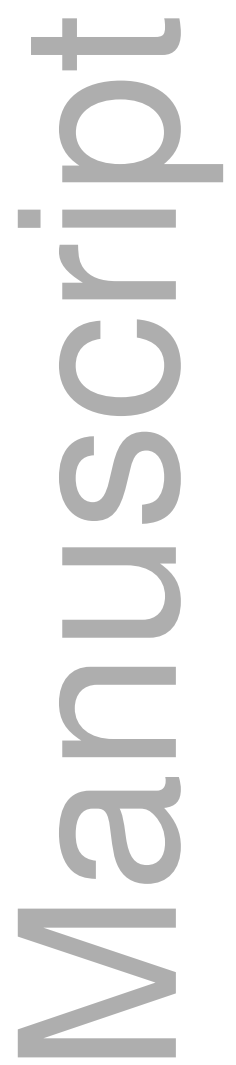

\begin{tabular}{|c|c|c|}
\hline & \multicolumn{2}{|c|}{ Hairs Collected } \\
\hline Patient \# & Placebo & Tyramine \\
\hline 1 & 3 & 12 \\
\hline 2 & 9 & 9 \\
\hline 3 & 8 & 14 \\
\hline 4 & 11 & 8 \\
\hline 5 & 16 & 11 \\
\hline 6 & 8 & 7 \\
\hline 7 & 42 & 17 \\
\hline 8 & 5 & 11 \\
\hline 9 & 44 & 36 \\
\hline 10 & 26 & 16 \\
\hline 11 & 66 & 39 \\
\hline 12 & 18 & 5 \\
\hline 13 & 31 & 12 \\
\hline 14 & 20 & 2 \\
\hline 15 & 10 & 6 \\
\hline 16 & 8 & 4 \\
\hline 17 & 20 & 4 \\
\hline 18 & 54 & 26 \\
\hline 19 & 85 & 82 \\
\hline 20 & 22 & 5 \\
\hline 21 & 62 & 57 \\
\hline 22 & 22 & 7 \\
\hline 23 & 55 & 58 \\
\hline 24 & 25 & 17 \\
\hline
\end{tabular}

Table 1. Number of hairs removed by brushing after using a shampoo containing tyramine versus placebo. 


\section{University Library}

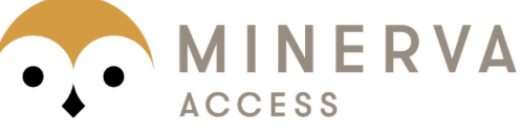

A gateway to Melbourne's research publications

Minerva Access is the Institutional Repository of The University of Melbourne

\section{Author/s:}

Kovacevic, M;McCoy, J;Goren, A;Situm, M;Stanimirovic, A;Liu, W;Tan, Y;Vano-Galvan, S;Shapiro, J;Sinclair, R

Title:

Novel shampoo reduces hair shedding by contracting the arrector pili muscle via the trace amine-associated receptor

Date:

2019-12-01

Citation:

Kovacevic, M., McCoy, J., Goren, A., Situm, M., Stanimirovic, A., Liu, W., Tan, Y., VanoGalvan, S., Shapiro, J. \& Sinclair, R. (2019). Novel shampoo reduces hair shedding by contracting the arrector pili muscle via the trace amine-associated receptor. JOURNAL OF COSMETIC DERMATOLOGY, 18 (6), pp.2037-2039. https://doi.org/10.1111/jocd.13054.

Persistent Link:

http://hdl.handle.net/11343/286109 